\title{
Size-dependent Thermotherapy of Iron Oxide Nanoparticles on Human Breast Adenocarcinoma Cancer Cells
}

Thikra Mustafa ${ }^{1}$, Yang Xu ${ }^{1}$, Fumiya Watanabe ${ }^{1}$, Yongbin Zhang ${ }^{2}$, Madhu P. Asar ${ }^{1}$, Reginald Little ${ }^{3}$, Alokita Karmakar ${ }^{1}$, Meena Mahmood ${ }^{1}$, Keith Hudson ${ }^{1}$, Alexandru S. Biris ${ }^{1}$

${ }^{1 .}$ Center for Integrative Nanotechnology Sciences, University of Arkansas at Little Rock, $2801 \mathrm{~S}$. University Ave, AR 72204, USA.

2. Nanotechnology Core Facility, Office of Scientific Coordination, National Center for Toxicological Research, US Food and Drug Administration, 3900 NCTR Road, Jefferson, AR, USA.

3. Department of Chemical Engineering, Howard University, 2366 Sixth St., Washington DC 20059, USA.

Hyperthermia is a promising approach for cancer treatment [1]. The optimal conditions for thermalization of RF energy by iron oxide nanoparticles (IONPs) are investigated in this study. The size and concentration of nanoparticles are among the most important parameters that have been studied for other nanoparticles [2-3]. These critical parameters should be optimized for IONPs applications in order to achieve the most efficient thermal transfer and, in turn, the highest efficiency for destroying cancer cells. Thus far, there have been few reports concerning the relationship between radio frequency and the size of the IONPs for RF-heating efficiency. The optimal particle size and concentration for thermalization of RF (two different frequencies) energy by IONPs are investigated in this study.

IONPs of 15, 25, and $41 \mathrm{~nm}$ in diameter used in this study were purchased from Ocean NanoTech (Springdale, Arkansas, USA). The size and shape of the particles were determined using a Transmission Electron Microscope (TEM), JEM-2100F (JEOL USA, Peabody, Massachusetts, USA) with an accelerating voltage of $80 \mathrm{kV}$ (Figure 1).

Human breast adenocarcinoma cancer cells (MCF-7) were used as a model to investigate the nanoparticle size-dependence of hyperthermia using two different frequencies of RF (350 kHz and $13.56 \mathrm{MHz}$ ). Different sizes of IONPs showed varying ability to destroy cancer cells under different frequencies of RF thermal activation. The $25 \mathrm{~nm}$ IONPs were found to be much more efficient in killing cancer cells compared to the other sizes at $350 \mathrm{kHz}$ low-frequency RF irradiation. However, at a higher RF of $13.56 \mathrm{MHz}, 15 \mathrm{~nm}$ IONPs produced the highest percentage of cell death. ROS release levels increased after RF treatment, which finally induced cellular apoptosis. TEM images of cells after RF exposure revealed that the cell plasma membranes had become uneven and that the cells showed signs of fragmentation by developing sealed membrane vesicles, termed apoptotic bodies (Figure 2). Moreover, scanning electron microscopy (SEM) revealed morphological alterations in the cell's plasma membrane, e.g., developing plasma membrane blebbing after RF exposure (Figure 3).

Our results will provide insight into how the sizes of IONPs influence their RF thermal energy delivery at different frequencies, which in turn will foster further research and development of magnetic nanoparticle-based hyperthermia treatments for cancer. 


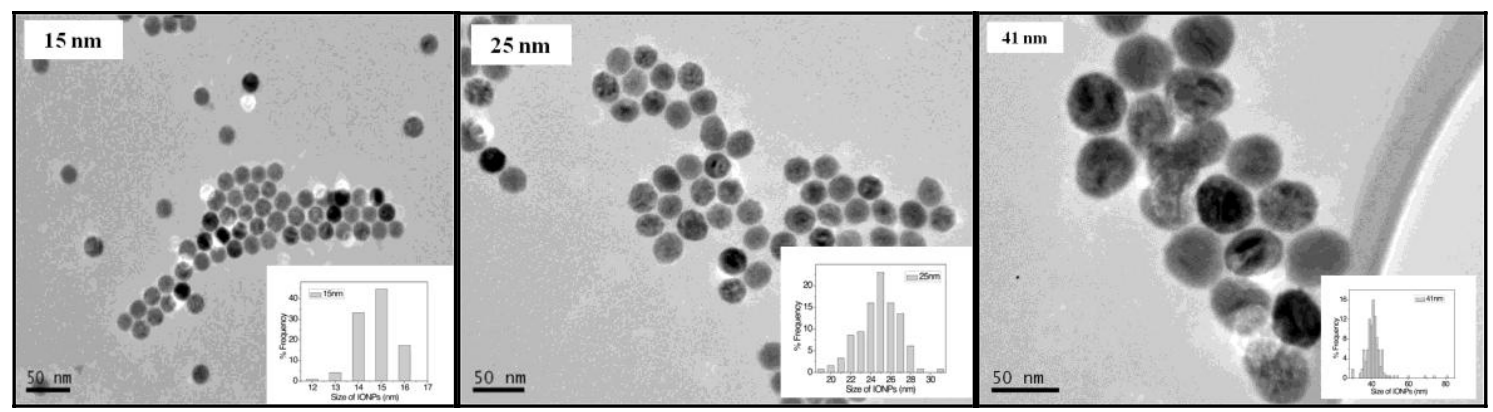

Figure 1. TEM images showing the morphology and size distribution graphs inset for the 15,25 , and 41 nm IONPs used in this study.

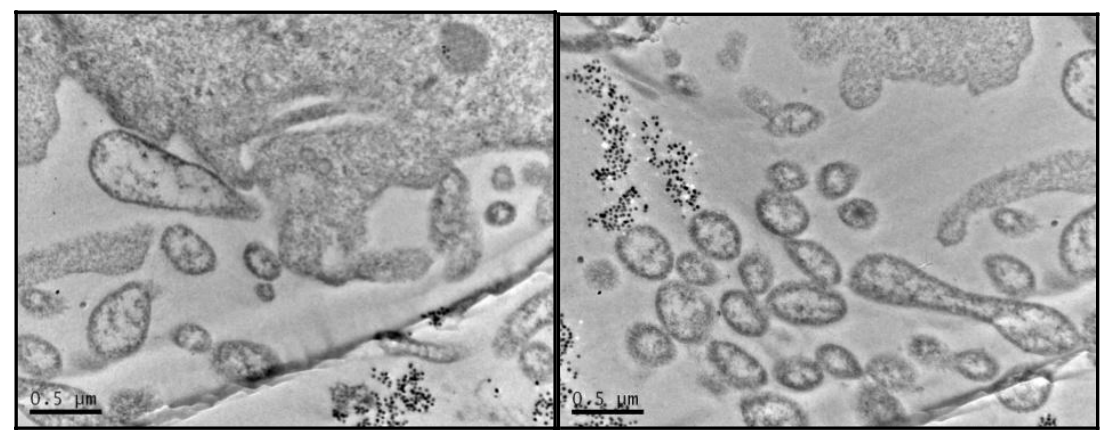

Figure 2. TEM images showing damging and fragmentation of $25 \mathrm{~nm}$ IONPs treated cells after RF exposure.

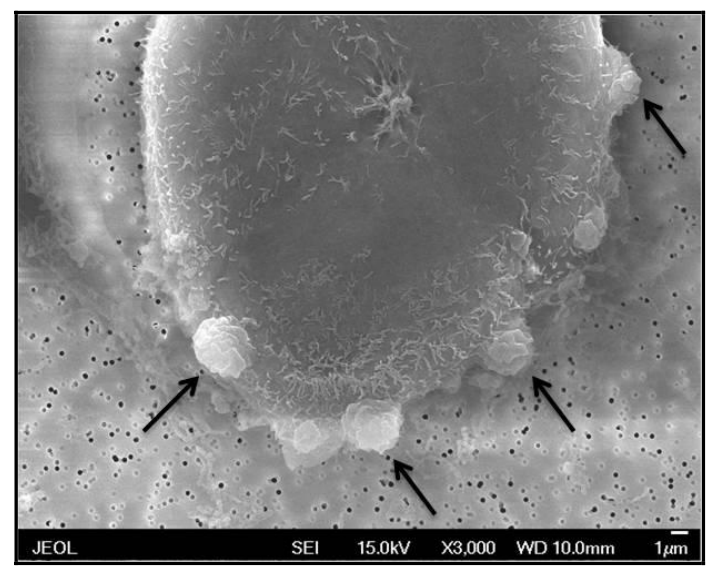

Figure 3. SEM image shows the outward evagination of MCF-7 cell plasma membrane after RF exposure.

\section{References}

[1] S. Krishnan, P. Diagaradjane and S. H.Cho, International Journal of Hyperthermia 26 (2010), 775. [2] C. L. Dennis, A. J. Jackson, J. A. Borchers, R. Ivkov, A. R. Foreman, P. J. Hoopes, R. Strawbridge, Z. Pierce, E. Goerntiz , J. WLau and C. Gruettner, J. Phys. D: Appl. Phys. 41 (2008), 134020.

[3] D.L. Zhao, H.L. Zhang, X.W. Zeng, Q.S. Xia and J.T. Tang, Biomedical Materials 1 (2006), 198. 